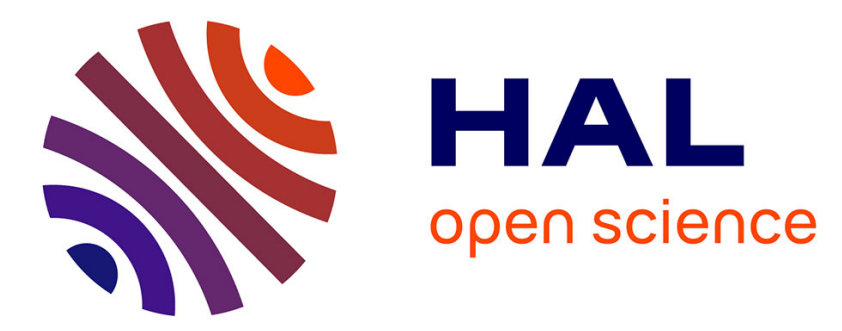

\title{
Identité basque et stratégie atlantique
}

Francis Jauréguiberry

\section{- To cite this version:}

Francis Jauréguiberry. Identité basque et stratégie atlantique. Sciences de la Société, 1995, 34, pp. 149-157. hal-00942992

\section{HAL Id: hal-00942992 \\ https://hal.science/hal-00942992}

Submitted on 6 Feb 2014

HAL is a multi-disciplinary open access archive for the deposit and dissemination of scientific research documents, whether they are published or not. The documents may come from teaching and research institutions in France or abroad, or from public or private research centers.
L'archive ouverte pluridisciplinaire HAL, est destinée au dépôt et à la diffusion de documents scientifiques de niveau recherche, publiés ou non, émanant des établissements d'enseignement et de recherche français ou étrangers, des laboratoires publics ou privés. 
Publié dans la revue Sciences de la société, n³ 34, Toulouse, 1995, pp. 149-157.

\section{IDENTITE BASQUE ET STRATEGIE ATLANTIQUE Francis Jauréguiberry*}

"Bilbao doit devenir la ville leader de ce que l'on appelle l'axe atlantique1. Il faut pour cela mobiliser avec dynamisme tout son potentiel humain, industriel et culturel et la doter des infrastructures qui correspondent au rôle de leader qu'on veut lui faire jouer. Pour cette tâche, les Institutions basques ${ }^{2}$ ont planifié des travaux d'infrastructure qui permettront à la Biscaye d'être le centre de la région atlantique qui part de Bordeaux et va jusqu'à la corniche cantabrique de la péninsule. Notre horizon immédiat se situe en 1997, date d'ici laquelle les Institutions publiques basques auront investi dans les seules infrastructures de l'aire métropolitaine de Bilbao environ 180000 millions de pesetas (9 milliards de francs)." Cette déclaration (11 mars 1992) de José Alberto Pradera Jauregui, Député général ${ }^{3}$ de la province de Biscaye, est la dernière en date que nous ayons relevée sur la façon dont les responsables des Institutions basques envisagent les rapports entre Euskadi et la Façade atlantique. Elle est à nos yeux bien significative du stade où l'idée d'axe atlantique est actuellement parvenue en Pays Basque : de référence purement discursive au départ, elle est en train de se muer en un véritable projet offrant un horizon européen adéquat à un ensemble d'actions et d'investissements.

Ayant vérifié que la plus grande partie de ces investissements auraient de toute façon été planifiés même si l'idée d'axe atlantique n'avait pas existé, notre attention s'est portée sur la question du statut référentiel de cet axe. S'agit-il d'un espace identitaire

\footnotetext{
* Maître de conférences de sociologie, chercheur au SET, Université de Pau et des Pays de l'Adour (avenue du Doyen Poplawski 64000 Pau). Ce texte est issu d'un travail réalisé pour la DATAR dans le cadre du programme "Prospective et territoires - Le devenir de la Façade atlantique".

${ }^{1}$ Les Basques font une nette distinction entre "axe atlantique" et "arc atlantique" (le terme de "façade atlantique" n'est jamais employé). L'arc atlantique renvoie à l'ensemble des régions de l'ouest européen, de l'Ecosse à l'Andalousie, et correspond à la Commission "Arc atlantique" de la "Conférence des Régions Périphériques et Maritimes" de la Communauté européenne. L'axe atlantique est une ligne nordsud allant de Paris à Madrid et passant par l'Aquitaine, la Navarre et le Pays Basque. Ces trois régions sont en train de multiplier leurs échanges et ont signé, le 13 février 1992, un protocole de coopération (celui-ci remplace deux protocoles antérieurement signés en octobre 1989 et septembre 1990 par les seules Aquitaine et Euskadi). L'ensemble Aquitaine-Euskadi-Navarre est considéré par les Basques comme une eurorégion d'équilibre et de développement.

2 Depuis son accession à l'autonomie (1979), le Pays Basque dispose d'institutions propres (60 000 fonctionnaires), d'un parlement et d'un gouvernement. Ce dernier (fait unique en Espagne) lève les impôts et verse chaque année un cupo (quote-part) au gouvernement central. Le budget du gouvernement basque est d'environ 28 milliards de francs. Ce gouvernement est responsable d'une police autonome, de la santé, de l'éducation, de la culture, de la recherche, de la planification territoriale, etc.

${ }^{3}$ Equivalent à un président de conseil général en France.
} 
dans lequel les Basques se situent spontanément pour penser et planifier leurs actions? Ou bien d'un espace d'extension, commode pour capter une richesse que le Pays Basque, pris isolément, ne possède pas mais que sa relative puissance (en regard de celle des régions avoisinantes) permet toutefois d'attirer? L'axe atlantique est-il vécu comme référence purement instrumentalisée, jouée en fonction des seules aides européennes escomptées? Ou s'agit-il, plus simplement encore, d'un espace de solidarité et de réaction de régions voisines que seul l'éloignement des axes dominants du développement européen rassemble?

\section{1- La non-pertinence professionnelle de l'axe atlantique pour les acteurs économiques}

La référence à l'axe atlantique est en Pays Basque moins le fait d'acteurs économiques que politiques. Hormis les responsables des Chambres de commerce et d'industrie, nous n'avons pas lu ou rencontré un seul dirigeant d'entreprise ayant fait preuve d'un réel intérêt, directement motivé par son statut professionnel, pour l'axe atlantique. Ces dirigeants se réfèrent avant tout à des réseaux (de fourniture, de savoir et de distribution), et la projection géographique de ces réseaux est loin d'être contenue dans les régions de la façade atlantique. Pire : ces réseaux sont bien plus denses en dehors d'elles qu'en leur sein! Pour les acteurs économiques rencontrés, l'axe atlantique est "une belle idée", un projet généreux, nécessaire pour obtenir la réalisation d'infrastructures inter-régionales et internationales jugées indispensables, mais répond d'une logique d'action dont ils "ne se sentent pas pour l'heure les principaux artisans dans la mesure où ce rôle est très bien tenu par les Institutions basques"4. Dans leur dimension intrinsèque d'entrepreneurs privés, les industriels ne se découvrent pas d'intérêts particuliers à privilégier l'axe atlantique. La seule retombée financière escomptée est celle de l'aide européenne au développement des régions de la façade atlantique sous forme de crédits pour l'investissement et la recherche, encore que le programme d'aide aux régions industrielles en déclin (dont bénéficie le Pays Basque) soit à leurs yeux beaucoup plus probant.

Par contre, en tant qu'acteurs économiques basques, c'est-à-dire s'identifiant fortement à un pays et à son devenir, ils produisent une analyse distincte renvoyant à un projet de développement collectif de la richesse en vue de puissance, et la Façade atlantique joue un rôle important dans ce projet. La prise en compte de l'axe atlantique par les acteurs économiques basques se fait donc par le biais non pas d'une lecture de type économiciste à partir de leurs intérêts immédiats et au moyen d'une logique

\footnotetext{
${ }^{4}$ Javier Hernández Bilbao, Secrétaire général de la Confédération du patronat basque (Confebask).
} 
utilitariste, mais de leur adhésion à un projet historique à partir d'une conscience identitaire et selon une logique politique de développement national ${ }^{5}$.

\section{2- La promotion basque de l'axe atlantique relève d'une action politique}

Si le thème de la "vertébration de l'axe atlantique" (pour reprendre une expression du Gouvernement basque) ne suscite chez les acteurs économiques qu'une adhésion seconde et non directement motivée par leur statut professionnel, il est par contre développé avec force et souvent même avec enthousiasme par les hommes politiques ayant en charge la gestion du Pays Basque. Il suffit de mesurer l'augmentation de la fréquence d'emploi du terme "axe atlantique" dans leurs discours et l'importance qu'ils lui accordent pour se rendre compte que cette idée est devenue référentielle en Pays Basque au moment de construire une stratégie de développement.

Cette stratégie peut être résumée ainsi : "L'espace atlantique européen, tant du côté français qu'espagnol, n'est actuellement qu'un relatif désert d'initiatives économiques, et souffre en conséquence d'une marginalisation en regard des grands axes européens et de leurs infrastructures en communication (...). En même temps, aucune des régions composant cet espace ne possède de façon isolée la force et le dynamisme suffisant pour générer par elle-même une culture économique et une créativité capables d'impulser un processus auto-accumulatif d'utilisation et de création des nouvelles technologies (que ce soit directement ou appliquées à des secteurs industriels traditionnels), pas plus qu'un poids politique suffisant pour convaincre les États français et espagnol ainsi que la Communauté européenne de la nécessité de mener une politique décidée de lutte contre l'isolement et la stagnation croissante de cet espace. Il existe donc les conditions objectives (identité des problèmes de base) et le champ d'action adéquat (évolution globale de l'Europe économique et politique) pour que soit non seulement nécessaire mais possible une action commune entre les régions de l'arc atlantique. Action destinée tant à encourager les facteurs endogènes de développement et l'apparition d'une nouvelle culture commune de modernisation, qu'à réclamer un appui exogène pour mener à bien cette tâche et pour combler les déficits en

\footnotetext{
5 L'expérience de deux des industriels que nous avons interviewés va même beaucoup plus loin. Pour eux, c'est essentiellement leur identification nationale qui explique la continuité de leur activité en Pays Basque. Une pure logique de rentabilisation du capital et d'expansion économique leur aurait en effet dicté, à la fin des années 70, de plier bagage et de s'implanter dans une autre région espagnole, à la fois politiquement plus calme et moins durement touchée par la crise. Ils admettent toutefois s'être alors posé la question "des limites au-delà desquelles le coeur conduit au suicide économique". Quelques années plus tard, les deux pensent avoir fait le bon choix, la situation du Pays Basque s'étant sensiblement améliorée à la fin des années 80 .
} 
infrastructures. C'est de la façon dont l'axe atlantique pris dans son ensemble répondra à ce défi que dépendra l'avenir de chacune de ses régions prises séparément"6.

Si l'on cherche à repérer quels sont les principaux promoteurs de cette stratégie atlantique, on s'aperçoit qu'ils sont tous, sans exception, affiliés ou très proches du Parti Nationaliste Basque (nationalistes modérés, démocrates-chrétiens et majoritaires), d'Eusko Alkartasuna (nationalistes modérés, sociaux démocrates et minoritaires) ou d'Euskadiko Ezkerra (nationalistes modérés, "nouvelle gauche" et minoritaires). Le Parti Socialiste (au pouvoir à Madrid mais minoritaire en Pays Basque), ainsi qu'Herri Batasuna (indépendantistes proches d'ETA) sont soit silencieux, soit dubitatifs? L'équation qui permet de comprendre l'adhésion d'acteurs économiques au "projet atlantique" (identification nationale = propension à appuyer l'idée d'axe atlantique) ne peut donc être mécaniquement appliquée aux politiques. Si le projet atlantique est en Pays Basque quasi exclusivement porté par des nationalistes, ce ne sont pas tous les nationalistes qui le défendent. Ses promoteurs sont des nationalistes qui s'opposent tant à l'État espagnol dans son désir centraliste qu'aux nationalistes radicaux qui prônent l'indépendance immédiate. Il importe donc de repérer plus précisément quel type de nationalisme conduit à un tel appui. $\mathrm{Y}$ répondre permettra de définir la nature stratégique de l'adhésion basque à l'axe atlantique et de tenter une évaluation prospective des chances de son succès.

Contrairement à ce que l'on peut par exemple observer en Aquitaine, le sentiment d'appartenance collective à une entité historique et territoriale est très fort en Pays Basque. Pour penser et délimiter l'espace de référence de gestion du social, les Basques, dans leur immense majorité, se réfèrent spontanément à un "même" dont les limites territoriales (si l'on excepte l'épineuse question de la Navarre et la référence plutôt romantique au Pays Basque français) correspondent à celles de l'actuelle Communauté autonome. Loin d'être un simple découpage administratif, comme nombre des régions françaises, cet espace est au contraire le fruit d'une histoire très différenciatrice dont un des fils conducteurs est une lutte ininterrompue depuis plus d'un siècle pour obtenir la reconnaissance institutionnelle du Pays Basque dans sa volonté d'autodétermination ${ }^{8}$. Le problème identitaire en Pays Basque réside donc moins dans la

\footnotetext{
${ }^{6}$ Gouvernement basque : Perspectivas desde el eje atlántico, Vitoria-Gasteiz, 1990, p. 114.

7 Aux dernières élections législatives basques (28 octobre 1990), l'ensemble des nationalistes modérés (dont la revendication historique est la reconnaissance du droit à l'autodétermination du Pays Basque) ont obtenu 47,06 \% des suffrages exprimés, les nationalistes radicaux (réclamant l'indépendance immédiate) $18,12 \%$, les socialistes (qui considèrent l'actuel statut d'autonomie comme la bonne et définitive solution au "problème basque") 19,61 \%, et la droite espagnole (qui s'accommode de l'actuel statut) 8,12\% .

8 Pour ce qui est de l'actualité, rappelons que, lors des quatre dernières élections, les nationalistes ont obtenus, toutes tendances confondues, une moyenne de 59 à $65 \%$ des voix. Pour une analyse sociologique du mouvement basque, voir Francis Jauréguiberry, Question nationale et mouvements sociaux en Pays Baque sud, thèse de doctorat, Ecole des Hautes Etudes en Sciences Sociales, 1983.
} 
définition et dans l'adhésion à ce "même" que dans la diversité des stratégies pour le faire vivre au regard d'autres identifications et obligations.

Pour les nationalistes modérés, souveraineté du Pays Basque et unité de l'Europe vont de pair. Le Parti Nationaliste Basque (créé en 1895 et dans lequel la quasi-totalité des nationalistes se sont reconnus jusqu'à ce que d'autres formations -en particulier ETA - apparaissent dans années 60) a toujours été l'avocat fervent d'une Europe unie. Ainsi, la première célébration (manifestation de 65000 personnes le 27 mars 1932 à Bilbao) de l'Aberri Eguna (jour de la patrie basque) a été convoquée sur le thème : "Un Pays Basque uni et souverain dans une Europe unie et souveraine". La nouveauté ne réside donc pas dans l'identification Euskadi-Europe qui est ancienne, mais dans le fait que ce lien n'ait plus, comme par le passé, besoin d'être idéologiquement défendu. Dans un climat pro-européen, elle peut désormais être présentée par les nationalistes sous la forme pragmatique d'une évidence historique et économique. C'est ainsi que l'on peut lire, dans une publication du Gouvernement basque : "À partir des tendances qui viennent d'être décrites (une analyse économique et politique de l'Europe de l'ouest précède), il est aisé de prévoir que la future délimitation des espaces économiques ne recouvrira pas l'actuelle carte des États avec leurs frontières bien dessinées, mais une nouvelle structuration du territoire européen à partir des régions, au sens large du terme. Or, l'étendue géographique de ces nouveaux territoires de solidarité ne correspond pas nécessairement à celle des actuelles administrations politiquement établies. Ces nouveaux territoires renferment en leur sein une série de fonctions et d'inter-relations qui définissent des problématiques et/ou des caractéristiques socio-économiques communes que ne prennent pas en compte les frontières actuellement établies" 9 . Ou encore : "Les nationalités et les régions d'Europe doivent être des protagonistes dans la construction européenne. Les propositions adoptées par l'Assemblée des Régions d'Europe en faveur du fédéralisme et du principe de subsidiarité comme pilier de l'unité de l'Europe, et d'autres mesures pour assurer la représentation des régions auprès des Institutions communautaires, constituent une bonne plate-forme face aux débats engagés au sein de la CEE"10.

En cédant certaines de leurs prérogatives à l'Europe, en effaçant leurs frontières, les États de la Communauté européenne sont en train de relativiser le concept de souveraineté étatique. Dans le même temps, des entités infra-étatiques à forte personnalité (Länders, régions, communautés autonomes) font pression sur les États dont ils relèvent pour obtenir une plus grande latitude de gestion. La souveraineté a

\footnotetext{
${ }^{9}$ Gouvernement basque : Perspectivas desde el eje atlántico, Vitoria-Gasteiz, 1990, p. 113.

10 José Antonio Ardanza (Président du Gouvernement basque), in Atlantica - Revue transfrontalière d'information régionale et européenne, $\mathrm{n}^{\circ} 1$, mai 1991, p. 8.
} 
tendance à s'échapper à la fois vers le haut (Europe) et vers le bas (Communautés régionales). S'appuyant sur cette analyse, le Gouvernement basque ne cesse de multiplier (en particulier avec ses homologues allemands des Länders, espagnols des Communautés autonomes historiques, italiens et belges des Régions et britanniques des Nations sans État ${ }^{11}$ ) ses interventions auprès des instances européennes afin que la région devienne circonscription électorale européenne, soit reconnue comme l'organe souverain de gestion le plus proche des citoyens, soit représentée au sein d'un Sénat des Régions, et puisse intervenir directement dans les organes européens de décision dès lors que ceux-ci traitent de questions l'intéressant directement.

Le Gouvernement basque se heurte (évidemment) en cela au Gouvernement espagnol qui pense exactement le contraire : "La pire manière de construire l'Europe serait de le faire à partir des régions"12. Le recours du Gouvernement central porté devant le Tribunal constitutionnel espagnol pour l'ouverture, par le Gouvernement basque, d'une représentation à Bruxelles, ou encore le malaise du même Gouvernement central devant la participation systématique du Gouvernement basque aux différents Comités, Commissions ou Conférences des Régions en Europe, illustrent bien ce désaccord.

\section{3- L'Europe permet de penser la réalisation de la nation autrement qu'à travers l'État}

Cette stratégie européenne permet aussi aux gestionnaires de la Communauté autonome de se démarquer des nationalistes radicaux sans pour cela renoncer à l'idée de souveraineté. Face aux indépendantistes qui sont contre l'État espagnol en vue de devenir un jour comme lui (souverain), les nationalistes modérés se font les défenseurs d'une Europe fédérale dans laquelle les régions seront à la fois autonomes et solidaires, et les États anachroniques.

Cette idée de l'anachronisme des États en Europe n'est pas, on l'a vu, nouvelle en Pays Basque. Mais elle prend peut-être un tout autre relief dans la situation historique actuelle définie en particulier par la signature du Traité de Maastricht et par l'accession à l'indépendance de nations en Europe centrale. Contrairement à ce que l'on pourrait penser spontanément, la fragmentation de certains États ex-communistes ne réjouit pas spécialement les nationalistes modérés. Pour eux, ce processus est non seulement potentiellement déstabilisateur de l'équilibre européen, mais anachronique : "Cet indépendantisme maintient l'automatisme et le quasi-fatalisme qui, jusqu'à il y a

\footnotetext{
11 Toutes ces régions participent aux travaux de l'Assemblée des Régions d'Europe (ARE).

12 Juan Manuel Eguiagaray, Ministre espagnol de l'Administration publique.
} 
bien peu, unissait indissolublement la Nation et l'État."13 Face à ce "vieux réflexe", le Président du Gouvernement basque constate que "l'Europe unie est en train de prendre une direction inverse : celle de la rupture de la relation Etat-nation à partir de la crise de la réalité même de l'Etat tel que nous l'avons connu ces deux derniers siècles"14. Analysant la relativisation de la souveraineté étatique, il "se demande si l'heure n'est pas venue en Europe de clore une période historique, qui a débutée avec la Révolution française, et au cours de laquelle l'État-nation fut (...) l'instrument d'implantation des grands principes de la démocratie libérale (...). Les États sont "fatigués" (...) et il est urgent de procéder aux profondes réformes de certains de leurs appareils qui se révèlent excessivement petits pour résoudre de grands problèmes (marchés économiques forts, défense, relations extérieures, etc.) et excessivement grands pour répondre aux demandes des citoyens (éducation, santé, services publics et sociaux, culture, etc.). Si une forme d'État doit se maintenir en Europe, ce ne sera certainement pas celle que nous avons connue jusqu'à présent d'État omnipotent et omniprésent, ni celle d'État voulant être à la fois nation."15

Il s'en suit que si le but des nationalistes reste toujours le même : la souveraineté nationale, les formes de son obtention et surtout de sa gestion changent. En conséquence, s'interrogent les dirigeants du Parti Nationaliste Basque, "dans une Europe où les concepts classiques de souveraineté, d'indépendance et de prérogatives étatiques sont en crise et commencent à être remplacés par d'autres qui parlent de solidarité, d'interdépendance et de participation (...), y a t-il encore quelque sens à continuer de définir notre stratégie politique autour des objectifs d'obtention d'un État propre et d'indépendance?"16. Cette analyse bouleverse profondément la définition de l'action nationaliste : "Les vrais nationalistes modernes sont non plus ceux qui luttent pour la réalisation de l'indépendance avec un outil usé (l'État), mais ceux qui construisent la région basque (dans sa puissance) en vue de sa pleine reconnaissance comme élément de la future Europe unie"17.

Cette volonté d'exister par-delà les États revêt pour le Gouvernement basque un double avantage. Elle permet d'une part de fondre dans une même pratique la gestion de l'actuelle Communauté autonome, la revendication nationaliste et une stratégie européenne, et d'autre part d'opposer ce discours et cette pratique aux indépendantistes qui discréditent selon lui la revendication nationale basque.

\footnotetext{
13 José Antonio Ardanza, Euskadi : cohésion nacional e integracion europea, discours du 6 mai 1992 à Santiago (Chili).

14 Ibidem

15 Ibidem.

16 PNV, Proclamación de candidatura para las elecciones al Parlamento Europeo, mars 1989.

17 Ibidem.
} 


\section{4- Eurorégion atlantique et leadership basque}

Le très actif appui basque à l'axe atlantique est sans conteste construit autour d'arguments de type économique. La prise de conscience de la marginalisation des régions de la façade atlantique en regard des grands axes du développement européen s'est traduite par une volonté d'union du Pays Basque avec ses voisins atlantiques afin de constituer un lobby suffisamment fort pour être entendu par Bruxelles, Paris ou Madrid au moment de la programmation des grands travaux d'infrastructure ou de la mise en place des actions d'aide au développement.

Cet appui est sans doute aussi motivé par le désir de retrouver une puissance perdue. Ce désir est mieux compréhensible si l'on ne perd pas de vue que le Pays Basque a été, sans discontinuité depuis près d'un siècle et jusqu'à la fin des années 70, la région espagnole à la fois économiquement la plus puissante d'Espagne (avec la Catalogne $)^{18}$ et politiquement la plus niée (et souvent réprimée) dans son désir d'autogestion. Les dirigeants basques ont fait l'analyse qu'ils ne retrouveraient pas à eux seuls la puissance d'antan. Ils possèdent par contre désormais d'institutions qui leur permettent de pouvoir espérer jouer un rôle de leader et surtout de capteur de richesses d'une nouvelle eurorégion.

Mais ces deux raisons, bien que très importantes, ne sauraient expliquer à elles seules pourquoi Euskadi est à l'origine de toutes les principales initiatives d'accords interrégionaux atlantiques, pourquoi elle est certainement la région qui appuie avec le plus de force et d'enthousiasme l'idée d'axe atlantique. Le détour que nous venons de faire par l'identité nationale permet d'avancer une autre explication à cet élan atlantique : l'axe atlantique est en train d'être converti par les dirigeants basques en espace de démonstration de leur nouvelle stratégie nationale au sein de l'Europe unie. Son succès n'est rien moins que politiquement et identitairement vital pour eux. Il s'en suit que l'axe atlantique est sûr de trouver en Pays Basque ses plus fidèles défenseurs.

\section{Références}

- BEAUCHARD Jacques, Devenir de la façade atlantique. Paris, DATAR, 1992

- GOBIERNO VASCO : Perspectivas desde el eje atlántico, Vitoria-Gasteiz, Departamento de economia y planificacion,1990

- GOBIERNO VASCO : Euskadi en la Comunidad europea, Vitoria, 1984

18 Il suffit de rappeler qu'il détenait avant la guerre civile plus de $50 \%$ du capital financier espagnol et que ses provinces ont été, entre 1955 et 1970, régulièrement classées première et seconde (sur les cinquante que compte l'Espagne) pour l'augmentation du PIB et pour le revenu par tête. 
- JAURéGUIBERRY Francis, Question nationale et mouvements sociaux en Pays Baque sud", thèse de doctorat, Ecole des Hautes Etudes en Sciences Sociales, 1983

- JAURéGUIBERRY Francis, "Nationalismo y violencia política", in PEREZ AGOTE Alfonso, Sociología del nacionalismo, Universitad del País Vasco, 1988

- FOURQUET françois, Le Pays Basque, l'Espagne et l'Aquitaine, Bayonne, Ikerka, 1994

- PEREZ AGOTE Alfonso, La reproduccion del nacionalismo. El caso vasco, Madrid, Siglo XXI, 1984

- SANTACOLOMA SANZ Juan Francisco et alii, Euskadi en el proyecto Europa, Madrid, Centro de estudios Ramon Areces, 1991.

\title{
Résumé
}

Bien qu'importants, les arguments de type économique ne sauraient à eux seuls expliquer pourquoi les dirigeants basques appuient avec autant de force l'idée d'axe atlantique. La prise en compte de leur stratégie de construction nationale permet de mieux comprendre cet appui. Cette stratégie (il en existe une autre, indépendantiste mais minoritaire) revient à encourager tout ce qui peut relativiser le poids actuel des États au profit d'une Instance européenne vers le haut, et de Communautés régionales vers le bas. En effaçant les frontières étatiques et en permettant à ses régions de développer une diplomatie, l'axe atlantique devient l'espace de démonstration de cette stratégie.

\section{Resumen}

Aunque sean importantes, los puros argumentos de tipo económico no podrían explicar porqué los dirigentes vascos apoyan con tanto fervor la idea del eje atlántico. La consideración de su estrategia de construcción nacional permite comprender mejor este apoyo. Esta estrategia (existe otra, independentista pero minoritaria) consiste en estimular todo lo que pueda relativizar el peso actual de los Estados a beneficio de una Instancia europea hacia arriba y de Copmmunidades regionales hacia abajo. Al borrar las fronteras entre Estados y al permitir a sus regiones desarrollar una diplomacia, el eje atlántico se convierte en el espacio de demonstración de esta estrategia.

\begin{abstract}
Economic arguments, although important, are not sufficient on their own to explain why Basque leaders insist so heavily on the idea of an Atlantic axis. An analysis of their strategy of national construction allows us to understand this approach more fully. This strategy (another independentist but minority one also exists) consists in encouraging everything which helps to relativise the actual weight of states in relation to a supranational European dimension above and regional communities below. By eliminating frontiers and allowing regions to develop their own diplomacy, the Atlantic axis has become the sounding board of this strategy.
\end{abstract}

